\title{
Dagmar Wieser, Nerval : une poétique du deuil à l'âge romantique
}

\section{Maria Rosaria Ansalone}

\section{Q OpenEdition}

1 Journals

\section{Edizione digitale}

URL: http://journals.openedition.org/studifrancesi/30578

DOI: $10.4000 /$ studifrancesi.30578

ISSN: 2421-5856

\section{Editore}

Rosenberg \& Sellier

\section{Edizione cartacea}

Data di pubblicazione: 1 avril 2006

Paginazione: 180-182

ISSN: 0039-2944

\section{Notizia bibliografica digitale}

Maria Rosaria Ansalone, «Dagmar Wieser, Nerval : une poétique du deuil à l'âge romantique», Studi

Francesi [Online], 148 (XLX | I) | 2006, online dal 30 novembre 2015, consultato il 19 avril 2021. URL: http://journals.openedition.org/studifrancesi/30578; DOI: https://doi.org/10.4000/studifrancesi. 30578

Questo documento è stato generato automaticamente il 19 avril 2021.

\section{(c) (1)}

Studi Francesi è distribuita con Licenza Creative Commons Attribuzione - Non commerciale - Non opere derivate 4.0 Internazionale. 


\title{
Dagmar Wieser, Nerval : une poétique du deuil à l'âge romantique
}

\author{
Maria Rosaria Ansalone
}

\section{NOTIZIA}

DAGMAR WIESER, Nerval : une poétique du deuil à l'âge romantique, Genève, Droz, ( "Histoire des idées et critique littéraire $\left.» n^{\circ} 412\right), 2004$, pp. 408.

1 Il lungo saggio di Dagmar Wieser, versione a stampa della tesi, sostenuta all'Università di Berna nel 1998 e diretta da Daniel Sangsue, percorre l'opera del Nostro e focalizza i luoghi nei quali si manifesta una denegazione della Morte - e ancor più un « deuil d'un objet d'amour» (p.17) - nelle sue variazioni tematiche, enunciative e stilistiche, secondo un approccio metodologico di poetica storica e di metapsicologia ispirata alle teorie freudiane (introduction, pp. 11-24).

2 Il volume si articola in due parti, la prima più breve suddivisa in quattro capitoli, la seconda in ben sette. All'agile conclusione fanno seguito le «Annexes» (pp. 376-380: essenzialmente l'elenco delle Odelettes di Nerval e la definizione della loro versificazione) ; una ricca bibliografia (pp. 380-95: divisa in cinque parti, le prime due Euvres citées e Monographies et articles consacrés à Nerval canoniche e pertanto indispensabili; le altre tre -Critique littéraire générale; Linguistique et philosophie du langage; Histoire, Philosophie, Psychanalyse - forse di solito sottese alla stratificazione critica di qualsivoglia lavoro che utilizzi adeguati strumenti interpretativi). Ultima Annexe, il sempre prezioso «Index des noms de personnes » copre le pp. 397-404.

3 La prima parte, «Le Mythe de l'écrivain endeuillé » (pp. 24-88), studia racconti e poesie che hanno come tema il lutto d'amore o familiare, mostrando come il mito stesso contribuisca a in/formare la distinzione tra prosa e poesia. Le «Figures de la privation » (cap. I, pp. 28-45), personali e collettive popolano le Promenades et Souvenirs, nonché Aurélia, dove infanzia e amore sono entrambi marcati dalla problematica della perdita. In particolare il motivo delle rovine custodisce il segreto funerario e induce 
una meditazione sulla caducità umana. Al sentimento di un esilio costitutivo delle origini, si sovrappone l'abbandono in cui versano luoghi monumentali, mentre l'eroenarratore diviene egli stesso incarnazione della perdita. Perdita della donna amata, perdita della madre, con - come unico contraltare - la creatività letteraria! La figura paterna, chiusa nel suo mutismo, incapace di costituirsi in modello, funge da "oppositore". Solo le leggende del « ciclo del Valois » possono garantire, così, il lavoro della memoria (cap. II, « Perte et volonté de savoir », pp. 46-54), e, affidate alle voci del territorio, al nonno, alle zie, a più lontani antenati, consentono di far risalire fino all'imperatore romano Nerva l'origine del nome e ricollocano nel passato archeologico fate e rappresentazioni femminili della madre mai conosciuta. Voci vive, memoria sonora, il canto e le canzoni folkloristiche del Valois riescono a dire quella morte della madre che il silenzio paterno castratore vorrebbe cancellare.

Tale raffigurazione del padre non ha, tuttavia, « rien d'absolument original » (p. 62, cap. III, «L'Écriture comme effraction », pp. 55-75) per Dagmar WIESER, che ne rintraccia la genesi in personaggi di Balzac, Stendhal, Heine, ma soprattutto nelle Confessions rousseauiane. Essa alimenterà poi pagine di Michelet o di Chateaubriand, prolungandone la discendenza fino alla contemporaneità: "des écrivains comme Claude Simon (L'Acacia, 1989), Jean Rouaud (Les Champs d'honneur, 1990) ou François Vigouroux (Grand-père décédé-Stop-viens en uniforme, 2000) ont construit des récits de filiation qui, longtemps après la guerre, s'avèrent tissés de "fables de deuil" ".

5 La riflessione romantica (Hugo, Mme de Staël) sulla teoria dei generi (lirico, epicotragico, drammatico), prefigurata già in Rousseau quando affermava l'anteriorità del canto poetico sulle forme della parola discorsiva, assume in Nerval una consistenza ontogenica : le convenzioni enunciative della poesia lirica autorizzano il «rêve » e la denegazione della finitezza, ché essa ripercorre le tre età del poeta, dagli entusiasmi della gioventù all'epoca degli amori a quella della disperazione. Febbre, follia, ritorno a una terra dei morti, alla madre, sono tutti alimenti che materiano l'ispirazione del Nostro, coniugandosi pure strettamente nel topos del Teatro, dove si mette in scena « une fantasmatique du deuil et de la réparation ».

6 La deuxième partie indaga le riscritture che concorrono a occultare la perdita originaria (cap. I, « Réécritures et occultation de la perte», pp. 91-121) : l'opera in prosa mette in scena i ricordi d'infanzia o li storicizza tramite miti e racconti, articolando indagine (contraddittoria) sulla figura della Madre e riflessione sul cronotopo (spazio - Paris vs le Valois - e tempo - racconto lineare vs acronie, sillepsi e presupposizioni), quale si configura nella finzione narrativa. Gli strumenti di analisi sono essenzialmente quelli della critica tematica (rivisitata, come quando si smentisce la tesi di Jean-Pierre Richard sul valore euforico del motivo dell'acqua in Nerval), dell'interpretazione psicanalitica e quelli della grammatica testuale e enunciativa, privilegiati, ad esempio da Jacques Bony. Di notevole interesse la disamina delle variazioni cui l'opera di Nerval sottopone i personaggi di Orfeo e soprattutto di Prometeo, anch'egli vittima del fuoco / della follia, piuttosto che capace di infrangerne l'interdetto!

7 Ma se il soggetto, nella coscienza diurna, appare capace di tornare al principio di realtà e scrive Aurélia (cap. II, "Théâtralité du deuil: l'exemple d'Aurélia», pp.122-150) a riprova delle sue capacità mentali e dell'avvenuta accettazione della perdita dell'amata, ben presto il disordine e il fantastico lo catturano e lo rivelano in preda al lutto dell'amore : le modalità verbali, lessicali innanzitutto, poi sintattico-testuali (discorso indiretto libero), dialoghi, riflessioni, commenti; sdoppiamento dell'enunciazione 
narrativa, modalizzazione e persino uso del trattino ("qui subdivise les scènes oniriques en séquences actancielles ou spatio-temporelles [et] revêt une fonction de 'découpage' cinématographique ", p. 135), confluiscono in quelle della macrostruttura del racconto, vanificando qualsivoglia tentativo di sintesi e di ricerca di un senso unitario. La denegazione della malattia mentale si sposa alla non-accettazione della morte di Aurélia e l'immaginazione, persa la connotazione romantica di forza energica e produttiva, diviene potenza compensatrice e alienante!

Il mestiere di romanziere, trasgressivo nei confronti del potere economico e politico (cap. III, "Mémoire familiale et dissidence politique» pp.151-189), deve affrontare censure e camuffare l'ispirazione autobiografica sotto il manto della tradizione realistica e storiografica: Angélique de Longueval, il Marquis de Fayolles, l'Abbé de Bucquoy o Léo Burckart sono altrettanti 'alter ego' del narratore che "établit une continuité entre les mémorialistes frondeurs et 'les écrivains' modernes ». Alla censura esterna si sovrappone quella interiore, e la lingua locale, parlata dialettale, unico strumento di rivendicazione delle origini e del passato, riafferma l'identità individuale e assicura la legittimazione esistenziale. Pura e trasparente « la langue des paysans » si contrappone al francese: orale, parola in senso saussuriano, popolare, la sua connotazione estetica si adatta a veicolare contenuti anticonformisti: il desiderio e la scrittura infrangono i lacci che inutilmente il paradigma paterno - repressivo, dominatore, monarchico - ha tentato di imporre a quello materno e femminile.

Il cap. IV, «Éros endeuillé, éros théurgique » (pp. 191-229), mostra come l'autobiografia spirituale degli Illuminés si iscriva nell'intento di rifiutare la finitezza dell'uomo: opera composita, essa riunisce l'alienato mentale (Spifane), come il credente (Cazotte, Bucquoy), come i seguaci dell'esoterismo (Restif, Cagliostro, Aucher), insomma tutti gli eccentrici della filosofia, capaci di sfuggire al regno del temporale attraverso la trascendenza divina o viceversa grazie alla via di fuga dell'immaginazione. Ma Les Illuminés vanno letti pure in relazione ai racconti personali, nella prospettiva, ancora una volta, del lutto d'amore e materno: una concezione ciclica e repressiva della temporalità induce la perdita e l'amore platonico impone la distanziazione dell'oggetto (l'attrice), fino a una vera e propria misoginia camuffata da imitazione del petrarchismo o della Vita nova di Dante. La fede nell'immortalità ha il solo scopo di permettere di rivedere 'la morta' e pone al suo centro il culto della dea Iside, capace di sconfiggere la paura della morte degli dei, grazie a un sincretismo intriso di scetticismo.

Poesia popolare, 'génie' primitivo del verso e della lingua, fascinazione della voce (a spese della musica e del senso delle parole), il progetto poetico di Nerval si nutre dell'opera di Ronsard e rifiuta la riforma di Malherbe: la struttura rigida del sonetto viene adottata al fine di disfare la rima classica ed enfatizzare l'orientamento tematico e spirituale dell'idea di un eterno ritorno. Tomba del Senso perduto, il sonetto delle Chimères (lunghissimo il cap. V, «Écritures en vers et refus de la finitude », pp. 230-311, quasi interamente dedicato a esse) diviene il simbolo stesso della memoria e continuamente evoca un'interlocutrice, divina o umana... Performativo, dialogico, il linguaggio poetico diviene teatrale, senza tuttavia mai conferire alle creature verbali evocate una concretezza oggettuale, mentre solo la poesia «chanson des recommencements, mémoire d'amour [...] semble pouvoir transcender un double deuil, cosmique et humain » (p. 260). 
11 Poco incline alla prosa poetica, espressione del passato magniloquente del Romanticismo ufficiale, Nerval respira Rousseau e Senancour (Un Roman à faire) e persino la prosa erratica del Diderot di Jacques le Fataliste (Les Nuits d'octobre), mentre segnala la distanza dalla prosa oratoria, sentimentale e lirica dei grandi del Romanticismo. Quanto al « poème en prose ", esso fa la sua apparizione nei Mémorables, 'ballata in prosa' che inserisce diversi sogni in Aurélia, racconto già di per sé onirico: vicino a Baudelaire, Nerval infrange le frontiere tra i generi, nella ricerca di un nuovo realismo critico, trasformando il racconto in un 'récit poétique' ante litteram per i criteri formali, per la spersonalizzazione dei personaggi, per il trattamento riservato alla temporalità, nonché per le sue esperienze di traduttore (soprattutto del Faust di Goethe).

12 Senza enfatizzare in maniera anacronistica l'hapax rappresentato dai Mémorables, D. Wieser sottolinea piuttosto l'inserimento ricorrente del verso nel racconto in prosa che, al cuore del "ciclo del Valois", viene proposto come ritorno alle origini della poesia francese, poesia "primitive», capace di " "rehausser la vieille versification française, affaiblie par les langueurs du XVIII ${ }^{\mathrm{e}}$ siècle" sans céder pour autant aux "brutalités des novateurs trop ardents"»(p. 294). Poetica del frammento, la voce indagata soprattutto nei suoi elementi soprasegmentali e prosodici, instaura una sinestesia che, in un percorso inverso a quello di uno Chateaubriand, ritrova la natura nel canto umano e non la voce dell'uomo nei suoni della natura... Voci delle nenie materne, variazioni del canto degli uccelli, suoni delle lingue straniere e sconosciute, altrettante sonorità predilette a tal punto da suggerire il fenomeno della metempsicosi, per il quale l'uomo si reincarnerebbe nella natura! Ed è così che « l'opposition entre la poésie et la prose tend à s'estomper. Nulle part ailleurs le récit nervalien se fait aussi “poétique" que lorsqu'il parle d'un chant » (p. 302). L'oscurità delle Chimères (si parla qui di « livre infaisable », tema che abbiamo studiato, cfr. M.R. Ansalone, "Angélique: la quête du livre et le livre infaisable », in "Cahiers de la Société Gérard de Nerval », $\mathrm{n}^{\circ}$ 10, 1987), annidata nella coesione enunciativa piuttosto che nell'impenetrabilità del sintagma (come avverrà invece in Mallarmé), rende queste composizioni poetiche teatro fantastico di una memoria che trova la sua realizzazione dialettica " dans le cadre resserré - “concentré" - du sonnet » (p. 306). Aprendo la poesia alla coscienza di sé, facendola accedere a una modalità della ragione in cui la ragione è assente, Nerval trova una via di salvezza per il suo lutto e scrive Aurélia.

Luogo di una sperimentazione infinita, la scrittura nervaliana si sforza di pervenire alla costruzione dell'io, testimoniata pure dalla costituzione della sua opera in " recueil ", con un volume che racchiude la sua produzione poetica, a sua volta inserito nel volume delle sue opere in prosa...

Nel breve cap. VI, « Dramaturgie de l'invisible » (pp. 312-32), il critico ricorda le circa venti opere teatrali, abbozzate o compiute, tra il 1827 e il 1855, e formula poi l'ipotesi che nei testi di natura teatrale si ritrovi l'ambizione costitutiva delle Chimères e cioè quella di «faire advenir une présence» (p.312), poiché «Poésie, théâtre, récit, nous retrouvons partout une logique du redoublement spéculaire. Illusion suprême, c'est à ce redoublement que Nerval demande de fonder dans la réalité la présence qui fuit » (p. 326). Critico teatrale, egli seppe indicare con sorprendente lucidità le ragioni dei suoi insuccessi nella sfortunata ricerca di nuove strade per il teatro (si veda al riguardo, tra l'altro, l'edizione di Léo Burckart e de L'Imagier de Harlem, per i tipi della Garnier Flammarion, a cura di Jacques Bony del 1996). 

fondamentalmente apologetica, quelle di viaggio si infittiscono nel momento della partenza e quando si avvicina il rientro, con un accentuarsi particolare del fenomeno in occasione dell'ultimo itinerario in Germania, dal quale il viaggiatore cerca di domare il dominante contrasto sulla sua carriera letteraria, riconoscendo al padre un vincolo con quella terra e con i ricordi materni a essa legati: « Les incursions en pays germanique se présentent dès lors comme un pèlerinage entrepris sur les traces du chirurgien d'armée » (p. 346) e la " Négociation de l'identité de l'écrivain » (p. 349) si annida fin nel paratesto, nella maniera di iniziare una lettera o di formularne l'indirizzo... Attanagliato da un inalienabile senso di colpa, il figlio tenta di delineare confluenze e paralleli col padre, persino nella tragica evidenza della sua malattia, sottomessa strenuamente al meccanismo della denegazione in un gioco sottile tra il detto e il nondetto, reso possibile da una vera e propria panoplia di artifici retorici. Preso nei lacci della relazione epistolare, egli diviene accusato e giudice al tempo stesso e finisce vittima dell'ossessione psicotica!

La conclusion contestualizza con una rapida 'esquisse' il mito dello scrittore in lutto o del lutto della scrittura nel Romanticismo francese, marcato dalle perdite successive della Rivoluzione, dell'Ancien Régime, dell'Impero... L'originalità di Nerval, rispetto ai suoi predecessori e successori letterari, risiede nel rinviare la lacerazione dell'io a una Trascendenza che si suppone capace di restituirgli unità, ma che appare sempre sul punto di sottrarsi all'individuo. Di fronte a tale impossibilità la sua opera si distoglie dal reale e si configura come tentativo (riuscito) di rievocazione. 